\title{
The efficacy of somatosensory evoked potentials in evaluating new neurological deficits after spinal thoracic fusion and decompression
}

\author{
Samyuktha R. Melachuri, BS,,2 Carolyn Stopera, BS, ${ }^{1,2}$ Manasa K. Melachuri, BS,1,2 \\ Katherine Anetakis, MD, 1,2 Donald J. Crammond, PhD, 1,2 James F. Castellano, MD, PhD, 1,2 \\ Jeffrey R. Balzer, PhD, ${ }^{1,2}$ and Parthasarathy D. Thirumala, MD, MS ${ }^{1,2}$ \\ Departments of ${ }^{1}$ Neurological Surgery and ${ }^{2}$ Neurology, University of Pittsburgh, Pennsylvania
}

\begin{abstract}
OBJECTIVE Posterior thoracic fusion (PTF) is used as a surgical treatment for a wide range of pathologies. The monitoring of somatosensory evoked potentials (SSEPs) is used to detect and prevent injury during many neurological surgeries. The authors conducted a study to evaluate the efficacy of SSEPs in predicting perioperative lower-extremity (LE) neurological deficits during spinal thoracic fusion surgery.
\end{abstract}

METHODS The authors included patients who underwent PTF with SSEP monitoring performed throughout the entire surgery from 2010 to 2015 at the University of Pittsburgh Medical Center (UPMC). The sensitivity, specificity, odds ratio, and receiver operating characteristic curve were calculated to evaluate the diagnostic accuracy of SSEP changes in predicting postoperative deficits. Univariate analysis was completed to determine the impact of age exceeding 65 years, sex, obesity, abnormal baseline testing, surgery type, and neurological deficits on the development of intraoperative changes.

RESULTS From 2010 to 2015, 771 eligible patients underwent SSEP monitoring during PTF at UPMC. Univariate and linear regression analyses showed that LE SSEP changes significantly predicted LE neurological deficits. Significant changes in LE SSEPs had a sensitivity and specificity of $19 \%$ and $96 \%$, respectively, in predicting LE neurological deficits. The diagnostic odds ratio for patients with new LE neurological deficits who had significant changes in LE SSEPS was $5.86(95 \% \mathrm{Cl} 2.74-12.5)$. However, the results showed that a loss of LE waveforms had a poor predictive value for perioperative LE deficits (diagnostic OR 1.58 [95\% Cl 0.19-12.83]).

CONCLUSIONS Patients with new postoperative LE neurological deficits are 5.9 times more likely to have significant changes in LE SSEPs during PTF. Surgeon awareness of an LE SSEP loss may alter surgical strategy and positively impact rates of postoperative LE neurological deficit status. The relatively poor sensitivity of LE SSEP monitoring may indicate a need for multimodal neurophysiological monitoring, including motor evoked potentials, in thoracic fusion surgery. https://thejns.org/doi/abs/10.3171/2019.12.SPINE191157

KEYWORDS posterior thoracic fusion; somatosensory evoked potentials; neurological deficit; intraoperative neurophysiological monitoring

$\mathrm{P}$ OSTERIOR thoracic fusion (PTF) is used as treatment for many pathologies, including trauma, deformity, tumor, and infection. However, PTF is associated with many possible complications intraoperatively, such as muscle denervation, ischemia, and revascularization injury. ${ }^{10,11}$ These complications are associated with long-term effects, including paraspinal muscle atrophy, scarring, decreased muscle strength, and chronic pain., ${ }^{9,13}$ During spi- nal surgery, intraoperative neurophysiological monitoring (IONM) with somatosensory evoked potentials (SSEPs) and transcranial motor evoked potentials (TcMEPs) serves to detect and prevent injury, thereby preserving baseline neural function. IONM monitors the function of the spinal cord, nerve roots, lumbar plexus, and adequacy of vascular supply to these elements. ${ }^{6,17-19}$ Our clinical studies and meta-analysis and systematic review of spinal procedures,

ABBREVIATIONS IONM = intraoperative neurophysiological monitoring; LE = lower extremity; PTF = posterior thoracic fusion; SSEP = somatosensory evoked potential; TCMEP = transcranial motor evoked potential; UPMC = University of Pittsburgh Medical Center.

SUBMITTED September 23, 2019. ACCEPTED December 31, 2019.

INCLUDE WHEN CITING Published online March 6, 2020; DOI: 10.3171/2019.12.SPINE191157. 
including anterior and posterior cervical fusion and idiopathic scoliosis, have indicated that significant changes in SSEPs are associated with increased risk of postoperative neurological deficits. ${ }^{17,19}$ Clinically significant changes in SSEPs, defined as a persistent $50 \%$ reduction in primary somatosensory cortical amplitude or a prolongation of response latency by $>10 \%$ from baseline, are used to alert the surgical team about impending neurological injury. These alerts, in turn, can guide further surgical interventions. ${ }^{19}$

The primary aim of this study was to evaluate the efficacy of SSEPs in detecting new lower-extremity (LE) neurological deficits by evaluating the specificity, sensitivity, and diagnostic odds ratio during PTF. The results of the study will underscore the importance of SSEPs as a neurophysiological surrogate for impending neurological deficits.

\section{Methods \\ Study Population}

All patients who underwent PTF with complete SSEP monitoring at the University of Pittsburgh Medical Center (UPMC) from 2010 to 2015 were included in this study. Please note that some of these surgeries extended to regions beyond the thoracic spine but were included because the bulk of the surgery was performed on the thoracic portion of the spine. SSEPs were recorded at baseline and determined to be within normal limits or to be abnormal; they were also used to determine the reliability for IONM itself. The institutional review board at UPMC approved this retrospective observational study.

\section{Neurological Deficit Criteria}

Medical records were reviewed before and after surgery to identify exacerbation of or new LE neurological deficits. Patients were considered to have a worsening or new neurological LE deficit if their postoperative examinations included sensory disturbances (e.g., hyperesthesia, paresthesia, numbness, or tingling), motor deficits (e.g., paresis, paraplegia or hemiplegia, or single nerve root palsies), or a combination of both as documented in the patients' charts.

\section{IONM}

\section{LE SSEPS}

The methods for SSEP positioning and recording are similar to those described in our previous publication ${ }^{17}$ for posterior cervical fusions. Please refer to that publication for the complete methods. Once each patient was put in the supine position, peripheral stimulating electrodes were bilaterally placed. Peroneal and tibial nerve stimulation was performed at the head of the fibula or the medial malleolus, respectively.

Three channels were recorded in response to LE stimulation: Pz-Fz and P3-P4 for cortical potentials and mastoid-Fz for brainstem/cervical potentials. The stimulation frequency was set to $2.33-2.41 \mathrm{~Hz}$ with a duration of $0.2-0.3$ msec. By increasing intensity until the cortical amplitude no longer increased with increasing stimulus intensity, the supramaximal stimulation intensity was determined. ${ }^{20}$

\section{Group Discrimination}

We calculated the sensitivity and specificity to assess the diagnostic accuracy of changes in LE SSEPs to identify postoperative LE neurological deficits. Patients were split into 3 categories: no changes in SSEPs, significant change in SSEPs, and loss of response in SSEPs. According to the American Society of Neurophysiological Monitoring and American Clinical Neurophysiology Society guidelines, the optimal sensitivity and specificity for detecting iatrogenic spinal cord injury are as follows: a consistent and persistent reduction of $>50 \%$ in primary somatosensory cortical amplitude, a prolongation of $>10 \%$ in latency from baseline, or a complete loss of response waveforms that persists for two consecutively completed SSEP averages. ${ }^{2,5,22}$ Loss of waveforms was a $100 \%$ loss of amplitude. Transient losses were waveforms that were lost and then recovered to baseline in amplitude and latency before the end of surgery. Persistent losses were SSEPs that did not recover before the end of surgery. Please refer to Fig. 1 for a visual representation of significant SSEP changes and loss of responses.

\section{Statistical Methods}

We calculated the mean and standard deviation of age, the proportion of patient sex, and percent incidence of new postoperative neurological deficits for all PTF surgeries. The specificity and sensitivity of SSEP categories (no change, significant change, and loss of response) were also calculated. A 95\% CI was determined for all sensitivities and specificities. We obtained the number of true positives, false positives, false negatives, and true negatives for each SSEP category. A true-positive test was a significant change in LE SSEPs with the presence of a new postoperative neurological deficit. A false-positive test was a significant change without new neurological deficits. No significant changes with new neurological deficits was considered a false negative. A true negative was no significant changes and no new deficit.

We used a receiver operating characteristic curve to further evaluate the diagnostic accuracy of SSEP changes. The area under the receiver operating characteristic curve, significance, and 95\% CI were calculated. Nonparametric distribution was used to calculate the standard error, and the significance for all tests was set at $\mathrm{p}<0.05$. All statistics were completed using SPSS version 24 (IBM Corp.). We utilized similar statistical methods to our publication on posterior cervical fusion surgery. ${ }^{17}$

\section{Results}

\section{Participants}

From 2010 to 2015, 771 eligible patients underwent SSEP monitoring during posterior thoracic surgery at UPMC. Of these 771 patients, $103(13.4 \%)$ also underwent TcMEP monitoring and 220 (28.5\%) underwent electromyography monitoring according to surgeon preference. The surgeries included 137 thoracic decompressions (17.8\%) and 535 spinal fusions (69.4\%). Twenty-three pa- 


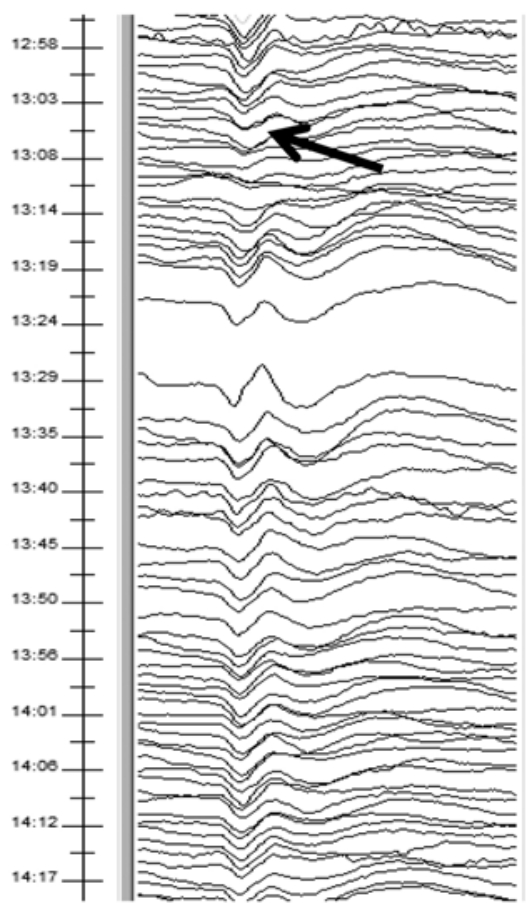

A: Significant changes

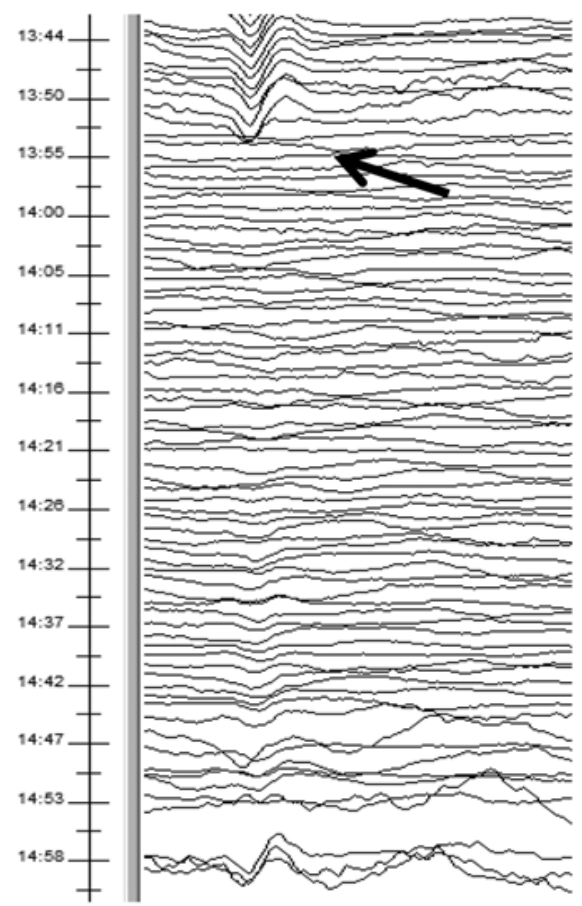

B: Transient Loss

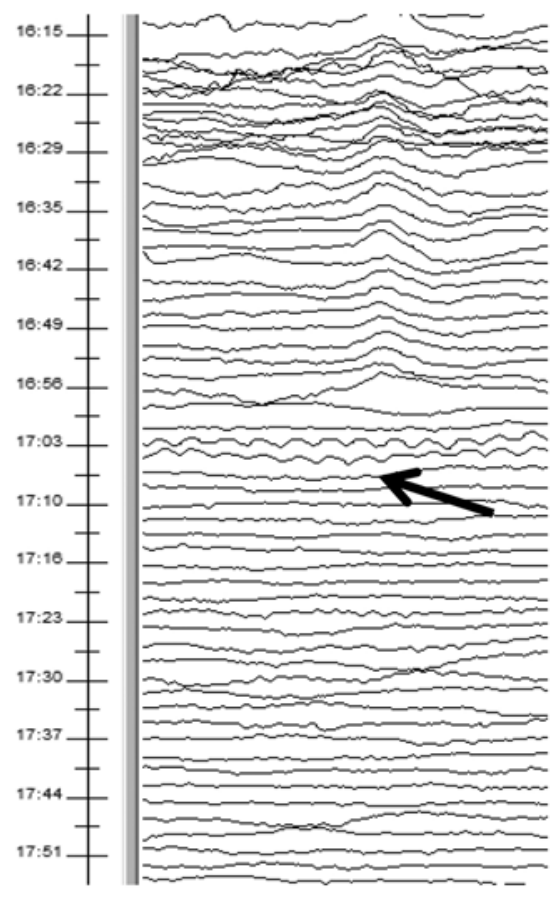

C: Permanent Loss

FIG. 1. Types of SSEP changes, including significant changes (A), a transient loss of response (B), and a permanent loss of response $(\mathbf{C})$.

tients who underwent spinal fusions also had decompressions. The type of spinal surgery for 52 patients (6.7\%) was inadequately documented. Please refer to Fig. 1 for the categorization of the patients in our study.

\section{Risk of Neurological Deficits}

Age, weight, sex, and previous medical conditions did not have a significant impact on whether the patient had LE SSEP changes intraoperatively. Type of spinal surgery also did not affect whether patients had LE SSEP changes intraoperatively. However, the results of our univariate analysis showed that patients with postoperative LE neurological deficits were more likely to have had significant changes in LE SSEPs. When adjusting for all the risk factors in multivariate linear regression analysis, postoperative LE neurological deficits also significantly predicted LE SSEP changes (Table 1).

\section{LE SSEPs and Neurological Deficits}

Of the 771 patients, 39 (5.06\%) had significant changes in LE SSEPs intraoperatively. Nine (23.1\%) of the 39 involved a loss of LE SSEPs, of which 7 (77.8\%) were a transient loss and 2 (22.2\%) were a persistent loss (Fig. 2).

A total of 57 patients $(7.39 \%)$ had new postoperative LE deficits. Eleven of these patients (19.3\%) with neurological deficits had significant LE SSEP changes, while 46 patients $(80.7 \%$ ) without significant LE SSEP changes had new LE neurological deficits, thus creating a relatively poor sensitivity rate of 0.19 (95\% CI $0.10-0.32)$. One patient $(14.3 \%)$ with a transient loss of SSEPs had a significant postoperative sensory neurological deficit.

\section{Description of LE Neurological Deficits}

The 57 patients with LE neurological deficits were characterized into categories based on the following type of deficit: sensory, motor, or sensorimotor. Twenty-eight patients (49.1\%) had sensory deficits, 21 (36.8\%) had motor deficits, and 8 (14\%) had sensorimotor deficits. LE sensory deficits included 23 patients $(82.1 \%)$ with numbness, 1 (3.6\%) with tingling, 2 (7.14\%) with numbness and tingling, and 2 (7.14\%) with paresthesia. LE motor deficits included 17 patients (81\%) with weakness and 4 (19\%) with paralysis. Eight patients experienced a combination of both motor and sensory deficits.

We then categorized patients into groups based on the following SSEP changes: no significant changes, significant changes, and loss of waveforms. Of the 28 patients, $20(71.4 \%)$ with sensory changes had no significant changes, while the remaining 8 patients $(28.6 \%)$ had significant changes in SSEPs. Eighteen (85.7\%) of the 21 patients with motor deficits had no significant changes, while the remaining 3 patients $(14.2 \%)$ had significant changes. None of the 8 patients (100\%) with sensorimotor deficits had significant SSEP changes.

\section{Results of Categorical Discrimination}

Table 2 provides a summary of the specificities and sensitivities of the types of LE SSEP changes. Using significant changes in LE SSEPs as the metric for the 771 patients, there were 11 true positives (1.43\%), 28 false positives (3.6\%), 686 true negatives (89.0\%), and 46 false negatives (6.0\%). Significant changes in LE SSEPs had a sen- 
TABLE 1. Results of univariate and multivariate analyses for patients with and without significant LE SSEP changes

\begin{tabular}{lccr}
\hline \multicolumn{1}{c}{ Variable } & LE SSEP Change & No LE SSEP Change & p Value \\
\hline No. of patients & $39(5.06)$ & $732(94.9)$ & \\
\hline Univariate analysis & & & \\
\hline Mean age \pm SD, yrs & $57.09 \pm 15.3$ & $56.97 \pm 15.3$ & 0.124 \\
\hline Female & $16(4.1)$ & $373(95.9)$ & 0.227 \\
\hline Hypertension & $17(5)$ & $320(95)$ & 0.893 \\
\hline Diabetes & $9(6.8)$ & $123(93.2)$ & 0.259 \\
\hline Coronary artery disease & $4(5.1)$ & $75(94.9)$ & 0.956 \\
\hline Tobacco use & $8(5.6)$ & $135(94.4)$ & 0.894 \\
\hline Obesity & $13(4.7)$ & $264(95.3)$ & 0.726 \\
\hline Abnormal baselines & $28(8.2)$ & $315(91.8)$ & 0.107 \\
\hline Decompression & $7(5.1)$ & $130(94.9)$ & 0.976 \\
\hline Spinal fusion & $27(5)$ & $508(95)$ & 0.982 \\
\hline LE neurological deficits & $11(19.3)$ & $46(80.7)$ & $<0.001$ \\
\hline Multivariate linear regression analysis & & & $<0.001$ \\
\hline LE neurological deficits & & & \\
\hline
\end{tabular}

Values are presented as the number of patients; parenthetical values are the percentage of patients in that particular category.

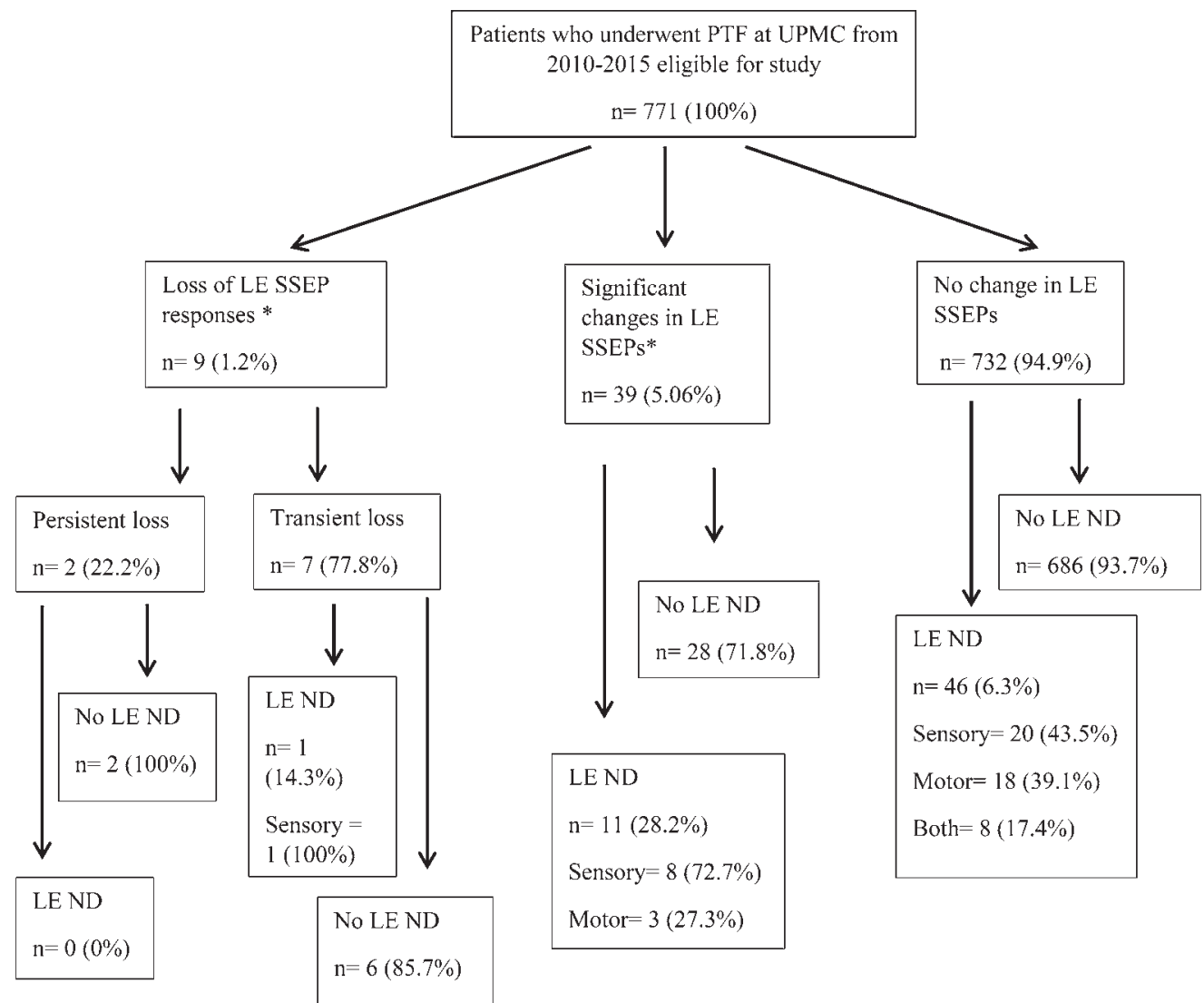

FIG. 2. Patient selection methods. Asterisk: Patients with LE loss of responses are included in the LE significant changes category. LE loss of responses is displayed separately in this figure to show the specifics of these patients. ND = neurological deficit. 
TABLE 2. Diagnostic accuracy

\begin{tabular}{|c|c|c|c|c|}
\hline Variable & $\begin{array}{c}\text { No Significant LE SSEP } \\
\text { Change }\end{array}$ & $\begin{array}{l}\text { Significant LE SSEP } \\
\text { Change }\end{array}$ & $\begin{array}{l}\text { Loss of LE SSEP } \\
\text { Waveforms }\end{array}$ & Total \\
\hline No. of patients & 732 & 39 & 9 & 771 \\
\hline Patients w/ ND & 46 & 11 & 1 & \\
\hline LE motor ND & 19 & 3 & 0 & \\
\hline LE sensory ND & 20 & 8 & 1 & \\
\hline Motor \& sensory NDs & 7 & 0 & 0 & \\
\hline Sensitivity $(95 \% \mathrm{Cl})$ & & $0.19(0.10-0.32)$ & $0.02(0.0-0.11)$ & \\
\hline Specificity $(95 \% \mathrm{Cl})$ & & $0.96(0.94-0.97)$ & $0.99(0.98-0.99)$ & \\
\hline Odds ratio $(95 \% \mathrm{Cl})$ & & $5.86(2.74-12.5)$ & $1.58(0.19-12.83)$ & \\
\hline AUC $(95 \% \mathrm{Cl})$ & & $0.61(0.51-0.71)$ & $0.51(0.33-0.68)$ & \\
\hline Youden's index & & 0.15 & 0.01 & \\
\hline
\end{tabular}

$\mathrm{ND}=$ neurological deficit.

sitivity and specificity of $19 \%$ and $96 \%$, respectively. Loss of waveforms had a sensitivity and specificity of $2 \%$ and $99 \%$, respectively. The area under the curve for significant change of LE waveforms was 0.61 (95\% CI 0.51-0.71) and that for loss of waveforms was 0.51 (95\% CI 0.33-0.68). The odds ratio for significant changes in LE SSEPs was 5.86 (95\% CI 2.74-12.5) and that for loss of responses was 1.58 (95\% CI 0.19-12.83).

\section{Discussion}

Our results show that SSEP monitoring during thoracic fusion procedures can predict new neurological deficits. Our work, in concordance with previous studies, supports the notion that SSEP monitoring alone can reduce postoperative paraplegia by $60 \%$ in spinal surgery. ${ }^{15,18}$ However, SSEPs can remain completely normal during nerve root injury, owing to central amplification, ${ }^{16,20,21}$ perhaps contributing to the relatively low sensitivity.

The authors of an IONM study in cervical spine surgery reported that SSEPs have a sensitivity and specificity of $52 \%$ and $100 \%$, respectively, in detecting postoperative neurological deficits. Kelleher et al. also determined that the return of SSEPs to baseline after a loss of responses predicts the absence of new deficits. ${ }^{12}$

Our results showed that significant changes in SSEPs during the thoracic fusion yield a very high specificity but a low sensitivity. A lack of significant changes in LE SSEPs is a good indication that there will be no neurological deficit. However, significant changes in LE SSEPs may not be the best predictor of new postoperative neurological deficits, which can result for many reasons. For example, deficits can develop postoperatively due to single dorsal root traction or kinking from spinal cord movement, ${ }^{1}$ which may not be sufficient to generate a significant amplitude change in LE SSEPs. The amplitude and latency of SSEPs can be affected by many factors, including halogenated agents, hypothermia, hypotension, and electrical interference. SSEP monitoring also does not detect root or motor deficits. ${ }^{16,21}$

The low sensitivity (19\%) of LE SSEPs for predicting a new LE deficit suggests that, in order to increase the sensitivity of IONM, additional IONM modalities such as TcMEPs should be added to SSEPs to enhance the IONM, since new motor deficits are largely not detected by SSEPs alone. ${ }^{12}$ Multimodal neuromonitoring utilizes the strengths of different types of IONM to optimize the diagnostic accuracy to detect impending neural injury. ${ }^{4,6,19}$ During corrective procedures, idiopathic scoliosis patients with new postoperative neurological deficits are 106.16 times more likely to have intraoperative SSEP and/ or TcMEP changes..$^{19}$ TcMEPs effectively monitor function of the corticospinal tract, while SSEPs monitor dorsal column function. TcMEP monitoring adds sensitivity for detecting new postoperative motor deficits. $., 7,8$

There are some limitations to our study that we would like to acknowledge. Our inclusion criteria included patients completely monitored with SSEPs. Whether these patients underwent other forms of intraoperative monitoring, such as TcMEP monitoring and electromyography, was noted, but not enough of the patients underwent these modalities to report our results as a multimodal study. Future investigations will be aimed at determining whether multimodal monitoring, namely SSEP and TcMEP, improves specificity for detection of postoperative neurological deficits. We did not monitor the progress of neurological deficits after hospital discharge; therefore, delayed deficits may have been omitted, and deficits that were present postoperatively may have been transient.

\section{Conclusions}

Patients with new postoperative LE neurological deficits are 5.9 times more likely to have significant changes in LE SSEPs during PTF surgery. Due to the low sensitivity of IONM using LE SSEPs alone, IONM sensitivity will increase by using a combined SSEP and TcMEP multimodality approach to detect perioperative LE neurological deficits in patients undergoing PTF.

\section{References}

1. Aita I, Hayashi K, Wadano Y, Yabuki T: Posterior movement 
and enlargement of the spinal cord after cervical laminoplasty. J Bone Joint Surg Br 80:33-37, 1998

2. Balzer JR, Rose RD, Welch WC, Sclabassi RJ: Simultaneous somatosensory evoked potential and electromyographic recordings during lumbosacral decompression and instrumentation. Neurosurgery 42:1318-1325, 1998

3. Bose B, Sestokas AK, Schwartz DM: Neurophysiological monitoring of spinal cord function during instrumented anterior cervical fusion. Spine J 4:202-207, 2004

4. Cheh G, Lenke LG, Padberg AM, Kim YJ, Daubs MD, Kuhns C, et al: Loss of spinal cord monitoring signals in children during thoracic kyphosis correction with spinal osteotomy: why does it occur and what should you do? Spine (Phila Pa 1976) 33:1093-1099, 2008

5. Chen ZY, Wong HK, Chan YH: Variability of somatosensory evoked potential monitoring during scoliosis surgery. J Spinal Disord Tech 17:470-476, 2004

6. Gonzalez AA, Jeyanandarajan D, Hansen C, Zada G, Hsieh PC: Intraoperative neurophysiological monitoring during spine surgery: a review. Neurosurg Focus 27(4):E6, 2009

7. Hilibrand AS, Schwartz DM, Sethuraman V, Vaccaro AR, Albert TJ: Comparison of transcranial electric motor and somatosensory evoked potential monitoring during cervical spine surgery. J Bone Joint Surg Am 86:1248-1253, 2004

8. Hsu B, Cree AK, Lagopoulos J, Cummine JL: Transcranial motor-evoked potentials combined with response recording through compound muscle action potential as the sole modality of spinal cord monitoring in spinal deformity surgery. Spine (Phila Pa 1976) 33:1100-1106, 2008

9. Jackson RK: The long-term effects of wide laminectomy for lumbar disc excision. A review of 130 patients. J Bone Joint Surg Br 53:609-616, 1971

10. Kawaguchi Y, Matsui H, Tsuji H: Back muscle injury after posterior lumbar spine surgery. A histologic and enzymatic analysis. Spine (Phila Pa 1976) 21:941-944, 1996

11. Kawaguchi Y, Matsui H, Tsuji H: Back muscle injury after posterior lumbar spine surgery. Part 1: Histologic and histochemical analyses in rats. Spine (Phila Pa 1976) 19:25902597, 1994

12. Kelleher MO, Tan G, Sarjeant R, Fehlings MG: Predictive value of intraoperative neurophysiological monitoring during cervical spine surgery: a prospective analysis of 1055 consecutive patients. J Neurosurg Spine 8:215-221, 2008

13. Kim DY, Lee SH, Chung SK, Lee HY: Comparison of multifidus muscle atrophy and trunk extension muscle strength: percutaneous versus open pedicle screw fixation. Spine (Phila Pa 1976) 30:123-129, 2005

14. Macnab IC, Cuthbert H, Godfrey CM: The incidence of denervation of the sacrospinales muscles following spinal surgery. Spine (Phila Pa 1976) 2:294-298, 1977

15. Nuwer MR, Dawson EG, Carlson LG, Kanim L, Sherman J: Somatosensory evoked potential spinal cord monitoring reduces neurologic deficits after scoliosis surgery: results of a large multicenter survey. Electroencephalogr Clin Neurophysiol 96:6-11, 1995
16. Owen JH, Padberg AM, Spahr-Holland L, Bridwell KH, Keppler L, Steffee AD: Clinical correlation between degenerative spine disease and dermatomal somatosensory-evoked potentials in humans. Spine (Phila Pa 1976) 16 (6 Suppl):S201S205, 1991

17. Thirumala PD, Melachuri SR, Kaur J, Ninaci D, Melachuri MK, Habeych M, et al: Diagnostic accuracy of somatosensory evoked potentials in evaluating new neurological deficits after posterior cervical fusions. Spine (Phila Pa 1976) 42:490-496, 2017

18. Thirumala PD, Bodily L, Tint D, Ward WT, Deeney VF, Crammond DJ, et al: Somatosensory-evoked potential monitoring during instrumented scoliosis corrective procedures: validity revisited. Spine J 14:1572-1580, 2014

19. Thirumala PD, Huang J, Thiagarajan K, Cheng H, Balzer J, Crammond DJ: Diagnostic accuracy of combined multimodality somatosensory evoked potential and transcranial motor evoked potential intraoperative monitoring in patients with idiopathic scoliosis. Spine (Phila Pa 1976) 41:E1177E1184, 2016

20. Tsai RY, Yang RS, Nuwer MR, Kanim LE, Delamarter RB, Dawson EG: Intraoperative dermatomal evoked potential monitoring fails to predict outcome from lumbar decompression surgery. Spine (Phila Pa 1976) 22:1970-1975, 1997

21. Tsai TM, Tsai CL, Lin TS, Lin CC, Jou IM: Value of dermatomal somatosensory evoked potentials in detecting acute nerve root injury: an experimental study with special emphasis on stimulus intensity. Spine (Phila Pa 1976) 30:E540E546, 2005

22. York DH, Chabot RJ, Gaines RW: Response variability of somatosensory evoked potentials during scoliosis surgery. Spine (Phila Pa 1976) 12:864-876, 1987

\section{Disclosures}

The authors report no conflict of interest concerning the materials or methods used in this study or the findings specified in this paper.

\section{Author Contributions}

Conception and design: Thirumala. Acquisition of data: SR Melachuri, Stopera, MK Melachuri. Analysis and interpretation of data: SR Melachuri, MK Melachuri. Drafting the article: SR Melachuri, MK Melachuri. Critically revising the article: Thirumala, SR Melachuri, Anetakis, Crammond, Castellano, Balzer. Reviewed submitted version of manuscript: Thirumala, SR Melachuri. Approved the final version of the manuscript on behalf of all authors: Thirumala. Statistical analysis: SR Melachuri. Study supervision: Thirumala.

\section{Correspondence}

Parthasarathy D. Thirumala: University of Pittsburgh, PA. thirumalapd@upmc.edu. 